\title{
Erythroid Differentiation of Induced Pluripotent Stem Cells Co-cultured with OP9 Cells for Diagnostic Purposes
}

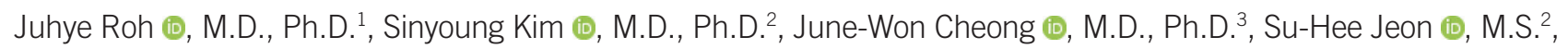

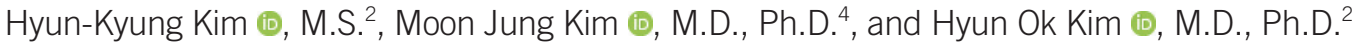

${ }^{1}$ Department of Laboratory Medicine, Hallym University Sacred Heart Hospital, Anyang, Korea; Departments of ${ }^{2}$ Laboratory Medicine and ${ }^{3}$ Internal Medicine, Yonsei University College of Medicine, Seoul, Korea; ${ }^{4}$ Department of Laboratory Medicine, Myongji Hospital, Goyang, Korea

Background: Reagent red blood cells (RBCs) are prepared from donated whole blood, resulting in various combinations of blood group antigens. This inconsistency can be resolved by producing RBCs with uniform antigen expression. Induced pluripotent stem cells (iPSCs) generated directly from mature cells constitute an unlimited source for RBC production. We aimed to produce erythroid cells from iPSCs for diagnostic purposes. We hypothesized that cultured erythroid cells express surface antigens that can be recognized by blood group antibodies.

Methods: iPSCs were co-cultured with OP9 stromal cells to stimulate differentiation into the erythroid lineage. Cell differentiation was examined using microscopy and flow cytometry. Hemoglobin electrophoresis and oxygen-binding capacity testing were performed to verify that the cultured erythroid cells functioned normally. The agglutination reactions of the cultured erythroid cells to antibodies were investigated to confirm that the cells expressed blood group antigens.

Results: The generated iPSCs showed stemness characteristics and could differentiate into the erythroid lineage. As differentiation progressed, the proportion of nucleated RBCs increased. Hemoglobin electrophoresis revealed a sharp peak in the hemoglobin $\mathrm{F}$ region. The oxygen-binding capacity test results were similar between normal RBCs and cultured nucleated RBCs. ABO and Rh-Hr blood grouping confirmed similar antigen expression between the donor RBCs and cultured nucleated RBCs.

Conclusions: We generated blood group antigen-expressing nucleated RBCs from iPSCs co-cultured with OP9 cells that can be used for diagnostic purposes. iPSCs from rare blood group donors could serve as an unlimited source for reagent production.

Key Words: Induced pluripotent stem cell, Blood group antigen, Cultured erythroid cells, OP9 cells
Received: May 13, 2021

Revision received: September 7, 2021

Accepted: January 23, 2022

\section{Corresponding author:}

Hyun Ok Kim, M.D., Ph.D.

Department of Laboratory Medicine,

Severance Hospital, Yonsei University

College of Medicine, 50-1 Yonsei-ro, Seoul

03722, Korea

Tel: +82-2-2228-2444

Fax: +82-2-313-0956

E-mail: hyunok1019@yuhs.ac

\begin{abstract}
(c) (1) $(9$
(c) Korean Society for Laboratory Medicine

This is an Open Access article distributed under the terms of the Creative Commons Attribution Non-Commercial License (https://creativecommons.org/licenses/by-nc/4.0) which permits unrestricted non-commercial use, distribution, and reproduction in any medium, provided the original work is properly cited.
\end{abstract}

\section{INTRODUCTION}

Providing compatible blood products to patients is essential for safe transfusion. In blood banks, pretransfusion testing is per- formed to determine the $\mathrm{ABO}$ and $\mathrm{Rh}$ types of red blood cells (RBCs) from patients and to screen serum for alloantibodies [1]. Pretransfusion testing not only ensures $\mathrm{ABO}$ compatibility between a donor and a patient but also detects clinically important 
alloantibodies that react with the donor RBC antigens [2, 3]. Reagent RBCs for pretransfusion testing, known as panel cells, are donated by healthy volunteers and are selected based on whether they carry the blood antigens necessary to detect the most clinically significant RBC alloantibodies. RBCs of different donors express different blood group antigens, resulting in inconsistencies in blood group antigens among reagent RBCs. Finding a suitable donor is especially difficult in the case of rare blood groups, and there is an insufficient supply of panel cells for rare blood group antigens. In vitro production of panel RBCs would improve the consistency of blood bank test results.

In the past decade, there have been extensive efforts to artificially produce human RBCs [4-9]. Hematopoietic stem and progenitor cells from the bone marrow and cord blood are used for RBC generation [10, 11]. However, these sources are suboptimal for large-scale production. Stem cells are another source for RBC generation. There are three types of stem cells: embryonic stem cells, adult stem cells, and induced pluripotent stem cells (iPSCs) [12]. Embryonic stem cells are pluripotent, meaning that they can differentiate into any cell type, but cannot generate a complete organ. Embryonic stem cells are difficult to obtain and use because of ethical issues, and they have the potential to differentiate into malignant tumor cells [13]. The differentiation ability of adult stem cells is lower than that of embryonic stem cells [14]. By contrast, iPSCs have the advantages of not being associated with ethical issues and capable of differentiating into any cell type. Once an iPSC line for a rare blood group is established and stored, it can be continuously used for cell culture.

The OP9 cell line, derived from mouse bone marrow stromal cells through a macrophage colony-stimulating factor gene mutation, has hematopoietic supporting capacity [15]. OP9 cells have been used as a feeder layer to support the in vitro differentiation of PSCs into various hematopoietic lineage cells and to increase the expansion potential of adult erythroid cells [16-18]. The efficiency of hematopoietic stem cell production can be increased via co-culture with OP9 cells, which may reduce the cost of in vitro RBC synthesis [19].

We aimed to generate blood group antigen-expressing erythroid cells from iPSCs co-cultured with OP9 cells. To the best of our knowledge, this is the first study to generate iPSC-derived erythroid cells for diagnostic purposes. We hypothesized that iPSC-derived erythroid cells have similar morphologies and functions as mature RBCs and express similar surface antigens capable of interacting with blood group antibodies.

\section{MATERIALS AND METHODS}

\section{Study design}

Three healthy volunteers with blood type $\mathrm{O}$, who provided informed consent, were enrolled in this study under authorization of the Institutional Review Board of Severance Hospital, Seoul, Korea (IRB number: 4-2018-0890). The study was conducted from March 2019 to June 2020. We generated iPSCs from peripheral blood mononuclear cells (PBMNCs) from the donors and differentiated them into erythroid cells (Fig. 1). To characterize the erythroid cells, we performed peripheral blood smear testing, blood group antigen phenotype testing, flow cytometry, and oxygen-binding capacity testing. The erythroid cells were treated with monoclonal RBC antibody reagents (Ortho Clinical Diagnostics, Raritan, NJ, USA). Data were collected for the human embryonic stem cell line H9 (WiCell, Madison, WI, USA) as a control, two newly derived iPSC lines from blood group O Dpositive donors, and one newly derived iPSC line from an O Dnegative donor.

\section{Generation of iPSCs}

To produce iPSCs, $10 \mathrm{~mL}$ of whole blood was drawn peripherally from the healthy donors. The antigen phenotype of the donor RBCs was determined using monoclonal RBC antibody reagents (Ortho Clinical Diagnostics). Ficoll-Paque (GE Healthcare, Uppsala, Sweden) was used to isolate mononuclear cells. Viable PBMNCs were counted using the trypan blue exclusion method ( $0.4 \%$ trypan blue stain, Gibco by Life Technologies, Carlsbad, CA, USA) and a hemocytometer [20]. The cells were resuspended and seeded in erythroid expansion medium and cultured until the proportion of erythroid progenitor cells among total cells reached $80 \%$; this was assessed using microscopy.

For transfection, the cells were suspended in medium comprising Nucleofector Solution (Lonza, Köln, Germany) and Epi5 Episomal iPSC Reprogramming Kit reagents (Thermo Fisher Scientific, Waltham, MA, USA) at room temperature $\left(20-22^{\circ} \mathrm{C}\right)$ for 10 minutes, per the manufacturer's instructions. The mixture was transferred into a Nucleocuvette vessel (Lonza) and loaded onto the 4D-Nucleofector system (Lonza). Electrotransfection was performed using the "CD34 cell, human cell type" program per the manufacturer's instructions. Human iPSCs were generated using a combination of reprogramming factors (OCT4, SOX2, KLF4, L-MYC, and LIN28). After transfection, the medium was changed to ReproTeSR Basal Medium (Stem Cell Technologies, Vancouver, Canada) and refreshed daily until iPSC colonies were observed. 


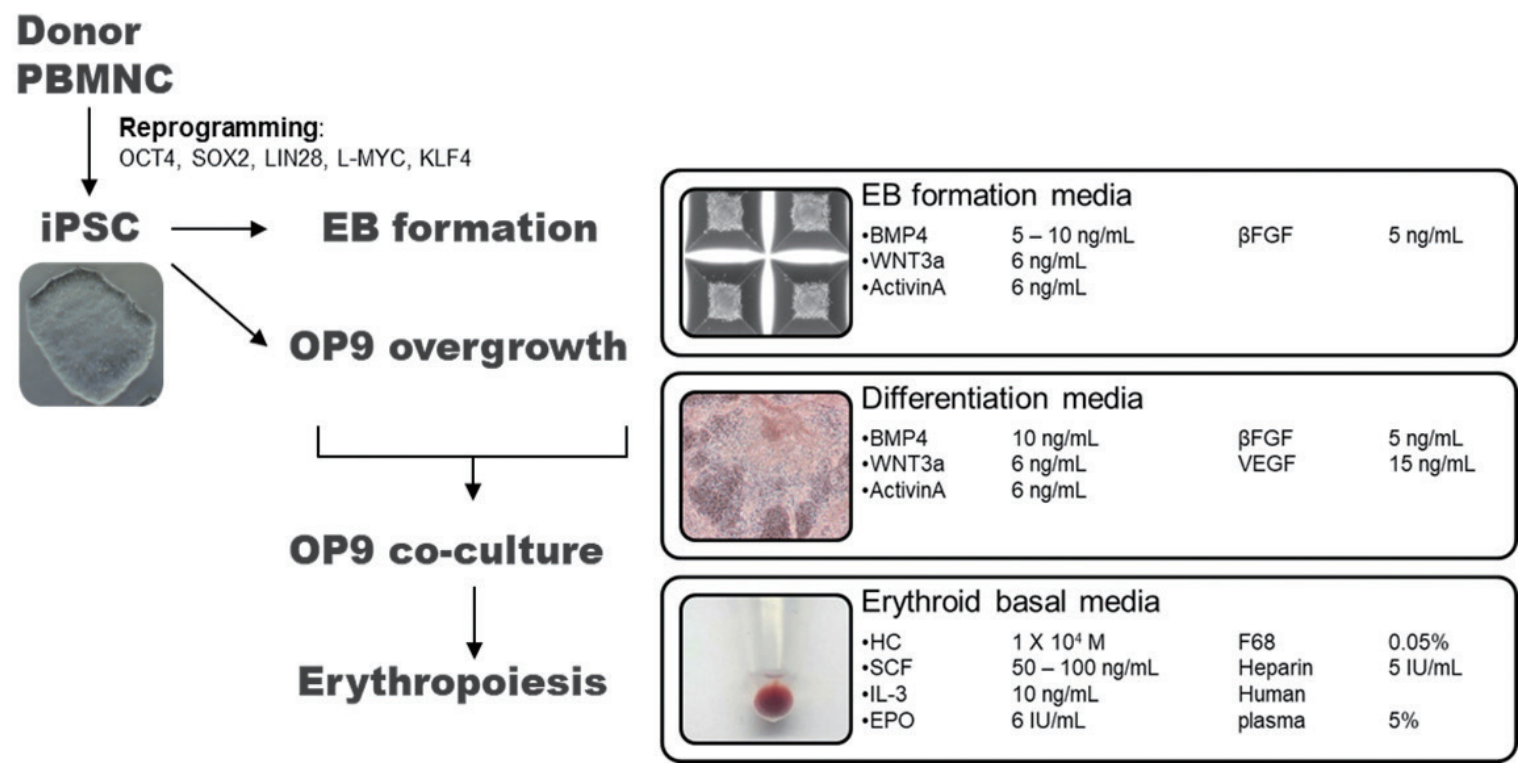

Fig. 1. Schematic representation of iPSC production and RBC differentiation. Human iPSCs were generated from PBMNCs via transfection with OCT4, SOX2, LIN28, L-MYC, and KLF4 (reprogramming factors). Erythroid differentiation was initiated after 8-10 passages. The process proceeded in three stages. First, embryoid bodies were formed to increase the differentiation efficiency. Second, the iPSC embryoid bodies were co-cultured with OP9 bone marrow stromal cells. Third, RBC differentiation was achieved using erythroid basal medium containing various cytokines.

Abbreviations: IPSC, induced pluripotent stem cell; PBMNC, peripheral blood mononuclear cell; RBC, red blood cell; BMP4, bone morphogenetic protein 4; WNT3a, wingless type 3a; $\beta F G F$, basic fibroblast growth factor; VEGF, vascular endothelial growth factor; HC, hydrocortisone; SCF, stem cell factor; IL-3, interleukin-3; EPO, erythropoietin.

\section{Karyotyping}

Genomic DNA was isolated from human iPSCs using the DNeasy Blood and Tissue kit (Qiagen, Hilden, Germany) per the manufacturer's instructions. Karyotypes were determined using the quantitative (q)PCR-based hPSC Genetic Analysis Kit (StemCell Technologies), which comprises primers for the eight most common karyotypic abnormalities observed in human iPSCs, and analyzed using the manufacturer's online genetic analysis application [21].

Detection of pluripotent germ layer markers in the established iPSCs

The iPSCs were maintained on 10-cm culture plates coated with $0.1 \%$ gelatin solution (GenDEPOT, Katy, TX, USA) in $10 \mathrm{~mL}$ of three-germ-layer differentiation medium (DMEM, 10\% fetal bovine serum; Gibco). The cells were cultured for two weeks, and the medium was refreshed once every 2-3 days. Total RNA was isolated from the cultured cells using the RNeasy Plus Mini Kit (Qiagen). cDNA was generated using the iScript cDNA Synthesis Kit (Bio-Rad, Hercules, CA, USA) and analyzed by reverse transcription (RT)-qPCR using the TaqMan Gene Expression Master Mix (Applied Biosystems, Foster City, CA, USA) on StepOnePlus Real-Time PCR System (Applied Biosystems). The following TaqMan probes (Applied Biosystems) were used: Brachyury, Hs00610080_m1_FAM; Sox17, Hs00751752_s1_ FAM; Nestin, Hs04187831_g1_FAM; and GAPDH, Hs02758991_ g1_VIC. GAPDH expression was used to normalize the data, and relative expression was calculated using the $\triangle \Delta C T$ method. The significance of differences in expression between samples was determined using Student's t-test.

iPSC differentiation into the erythroid lineage

An embryoid body was induced using AggreWell EB formation medium (StemCell Technologies). Three days after incubation in AggreWell, the embryoid body was placed on a layer of confluent adherent OP9 cells. After microscopically confirming the wheel shape of cells co-cultured for eight days, the cells were collected using collagenase IV and trypsin/EDTA.

On day 1 of differentiation to the erythroid lineage, a singlecell suspension of eight-day-co-cultured cells was prepared in erythroid basal medium containing Stemline II, $150 \mu \mathrm{g} / \mathrm{mL}$ human transferrin, $50 \mu \mathrm{g} / \mathrm{mL}$ insulin, $90 \mathrm{ng} / \mathrm{mL}$ ferric nitrate nonahydrate, and $160 \mu \mathrm{M}$ monothioglycerol solution (all from SigmaAldrich, St. Louis, MO, USA). The medium was refreshed once every 2-3 days. Cell morphology was evaluated using cytocentrifugation (Cytospin 4, Thermo Fisher Scientific; 700 rpm for 7 
min) and Wright-Giemsa staining (Sigma-Aldrich).

\section{Characterization of cultured cells}

Flow cytometry was performed using a BD FACSVerse instrument (BD Biosciences, Franklin Lakes, NJ, USA) and data were analyzed using FlowJo software (version 10, Becton, Dickinson and Company, Ashland, OR, USA) per the manufacturer's instructions. For each sample, at least 100,000 events were acquired.

A Hemox-Analyzer instrument (TCS Medical Products Division, Southampton, PA, USA) was used for oxygen-binding capacity testing. The sample vial was filled with HEMOX-solution, additive-A, and anti-foaming agent (all from TCS). Freshly drawn blood was added to the vial, which was placed in the sample intake tube of the cuvette.

For hemoglobin electrophoresis, erythroid cells $(200 \mu \mathrm{L})$ in an EDTA bottle were prepared using the Capillary 2 system (Sebia, Paris, France) per the manufacturer's instructions. The Capillary 2 system is based on the principle of capillary electrophoresis, in which charged molecules are separated on the basis of their electrophoretic mobility, electrolyte $\mathrm{pH}$, and electro-osmotic flow. Sample quality was monitored using commercial $\mathrm{Hb} \mathrm{A} 2$ and $\mathrm{Hb}$ AFSC (Sebia) controls (comprising normal hemoglobins $\mathrm{A}$ and $\mathrm{F}$ and abnormal hemoglobins $\mathrm{S}$ and $\mathrm{C}$ ).

\section{Confirmation of blood group antigen}

ABO blood group testing was performed using Ortho BioVue cassettes (Ortho Clinical Diagnostics) composed of six columns. The first and second columns were coated with anti-A antibody and anti-B antibody, respectively. The third column was coated with anti-RhD antibody, which is a major component of the Rhblood group system. The fourth column was used for the auto control. The fifth and sixth columns, which are developed for serum typing, were not used as they were not needed for this study. To minimize the effect of the erythroid basal medium, erythroid cells were washed with $0.9 \%$ normal saline.

To confirm Rh-Hr blood group antigen expression by the erythroid cells, $\sim 0.3 \%$ of the $50 \mu \mathrm{L}$ erythroid cell sample in normal saline was loaded on top of each column of the Ortho BioVue System Rh grouping cassette (Ortho Clinical Diagnostics). For the $\mathrm{Rh}-\mathrm{Hr}$ grouping cassette, each column was coated with anti-C, anti-E, anti-c, and anti-e, respectively. After loading, the cassette was centrifuged (Ortho Biovue Centrifuge, Ortho Clinical Diagnostics; 800 rpm for 2 minutes followed by 1,500 rpm for 3 minutes). The results were read according to the position of the red line on the column; a red line located near the bottom of the column was read as negative, whereas a red line located near the top of the column was read as positive [22].

\section{RESULTS}

\section{Establishment of iPSCs}

A small colony of human iPSCs formed 10 days after transfection and was cultured for 4-5 passages (Supplemental Data Figure S1). RT-qPCR, flow cytometry, and immunocytochemistry were used to examine the stemness characteristics of the iPSCs, confirming that all generated iPSCs were pluripotent (Fig. $2 A-C$ ). The karyotypes of the three iPSC lines were normal, and they all showed pluripotency to differentiate into the three germ layers (Fig. 2D, E). RBC differentiation was initiated using iPSCs at passages 8-10.

\section{Characterization of cultured erythroid cells}

Cells co-cultured with OP9 cells were analyzed by flow cytometry on days $0,7,14$, and 21 of differentiation to detect the expression of hematopoietic and erythroid markers (CD34, CD43, CD31, CD36, CD235a, CD71, CD15, and KDR; CD15 and KDR were checked only on day 0 ). On day 0 of differentiation, cells positive for KDR, an endothelial progenitor marker, were observed, whereas the cells were negative for the myeloid marker CD15. CD34 and CD43 expression indicates a hematopoietic stem cell phenotype. CD31 and CD36 are markers of early erythroid progenitors, whereas CD235a and CD71 are markers of late erythroid cells. After differentiation, the phenotype shifted from that of hematopoietic stem cells to that of RBC precursors (Fig. 3).

During erythroid differentiation, the cells were subjected to Wright-Giemsa staining and observed under a microscope (Fig. 4). As of day 10 of differentiation, we observed proerythroblasts and basophilic erythroblasts. On day 19 of differentiation, the erythroid cells became more mature (Fig. 5A), and polychromatic and orthochromatic erythroblasts were observed. Differential cell counts revealed that proerythroblasts and basophilic erythroblasts were present in the early stage and were progressively substituted with polychromatic and orthochromatic erythroblasts as differentiation progressed.

Hemoglobin electrophoresis of the erythroid cells revealed a sharp peak in region 2 (Fig. 5B). Based on comparison with the reference peak, region 2 corresponded to $\mathrm{Hb}$ F. Fig. $5 \mathrm{C}$ shows the oxygen-hemoglobin dissociation curves; there were no significant differences in oxygen binding between normal and iPSC-derived nucleated RBCs (nRBCs). 
Roh J, et al.

iPSC-derived erythroid cells for reagents

(A)

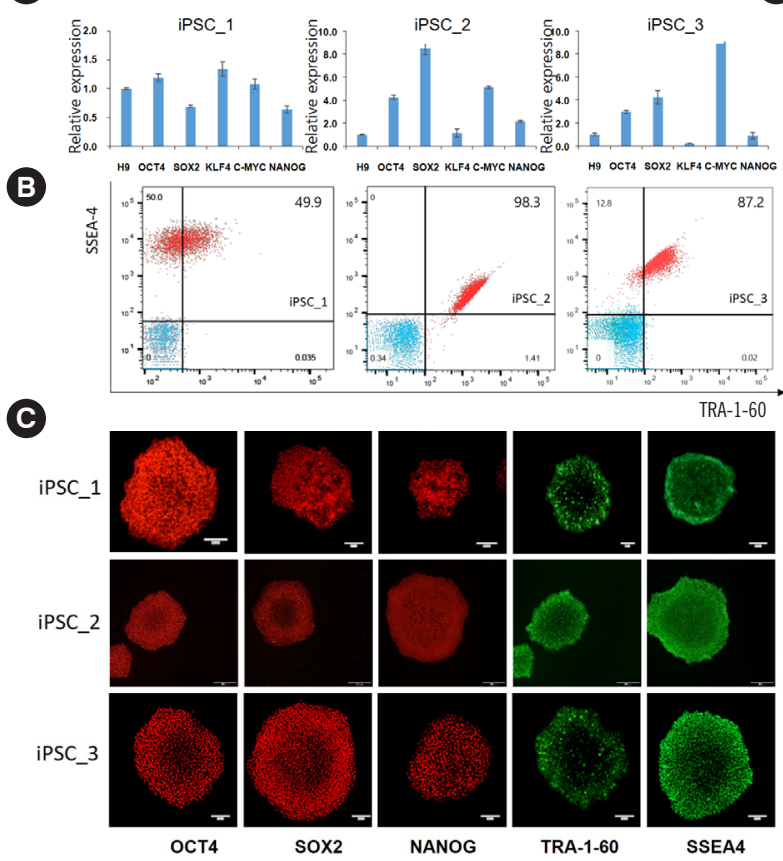

(D)
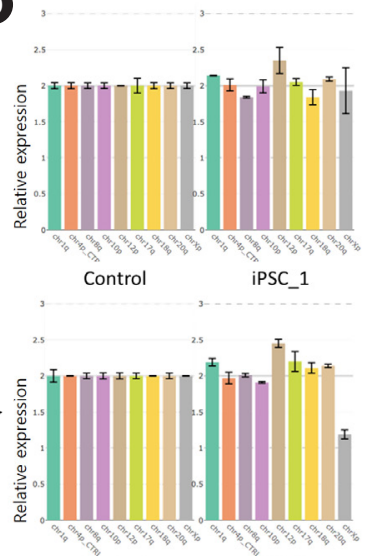

$\boldsymbol{E}$
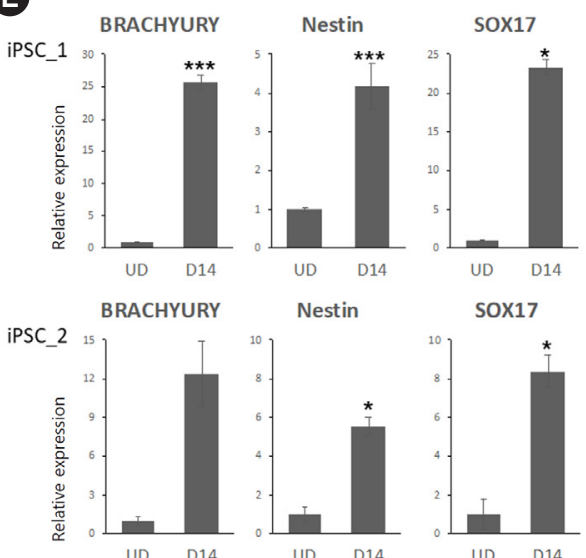

Nestin

Sox17
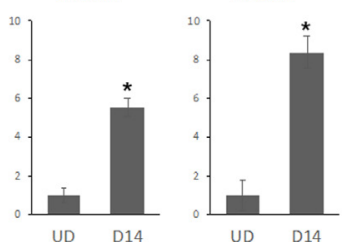

BRACHYURY

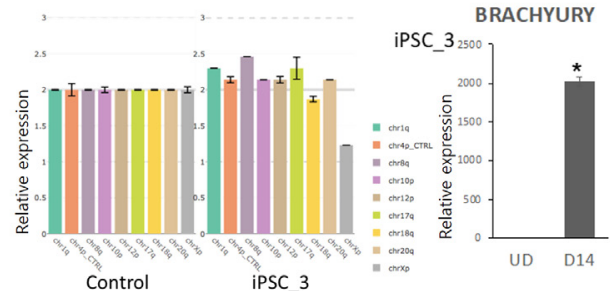

Sox17

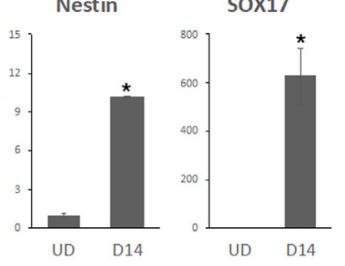

Fig. 2. Stemness characterization of iPSC lines generated from three donors (iPSC_1-iPSC_3). (A) RT-qPCR revealed that reprogramming factor genes were successfully transfected. (B) Flow cytometry and (C) immunocytochemistry staining revealed that each iPSC line expressed embryonic stem cell markers (scale bar $=100 \mu \mathrm{m}$ ). (D) Karyotyping indicated that the three iPSC lines had normal karyotypes at the eight most common karyotypic abnormality sites (chr1q, chr4p as a control, chr8q, chr10p, chr12p, chr17q, chr18q, chr20, and chrXp). Error bars indicate the range for the calculated copy number of the three replicates for each sample. (E) Detection of markers of the three germ layers verified that the iPSC lines showed pluripotency. Each gene expression level was compared before differentiation (UD) and on day 14 of differentiation (D14) using Student's t-test. ${ }^{*} P<0.05,{ }^{* * *} P<0.001$.

Abbreviations: IPSC, induced pluripotent stem cell; RT-qPCR, reverse transcription-quantitative PCR; UD, undifferentiated.

A
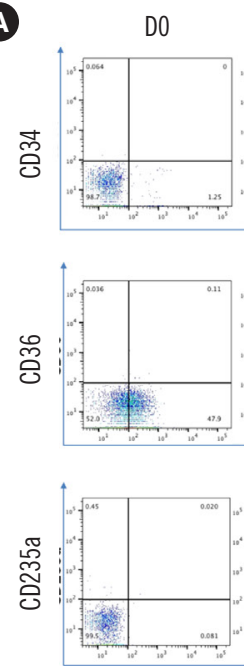

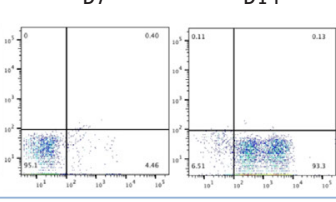

CD43

D7

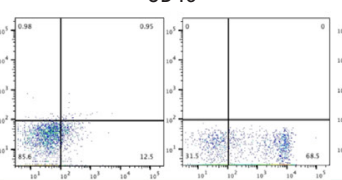

CD71

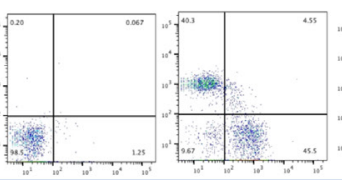

CD45
D21 B
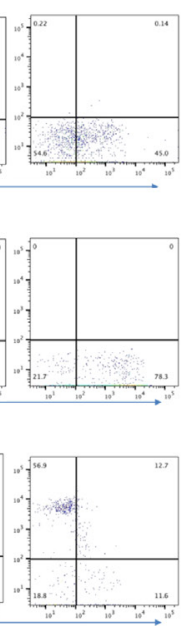

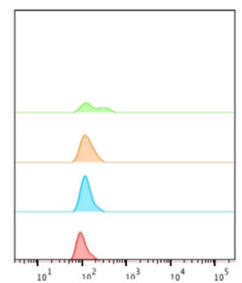

CD34

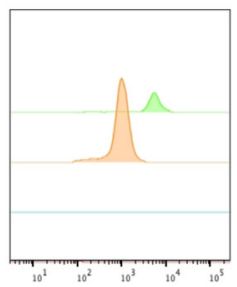

CD235a

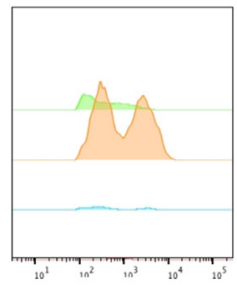

$\mathrm{CD} 43$

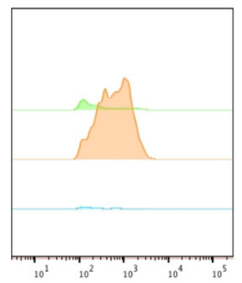

$\mathrm{CD} 45$

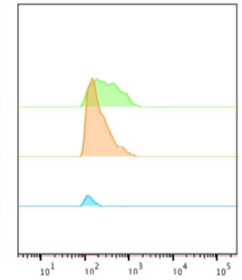

CD36

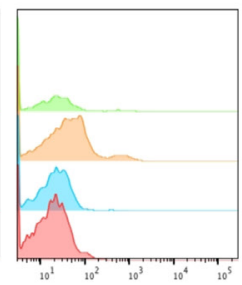

CD31

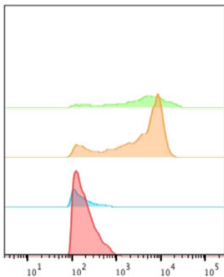

CD71

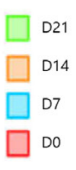

Fig. 3. Flow cytometry analysis of erythropoiesis. (A) The stem cell marker CD43 was expressed at the beginning of differentiation, and its expression level decreased as differentiation progressed. Similarly, the expression level of the early erythroid progenitor marker CD71 decreased. In contrast, the expression level of the late erythroid progenitor marker CD235a increased as differentiation progressed. (B) Expression of the different markers according to the day of differentiation. 
A

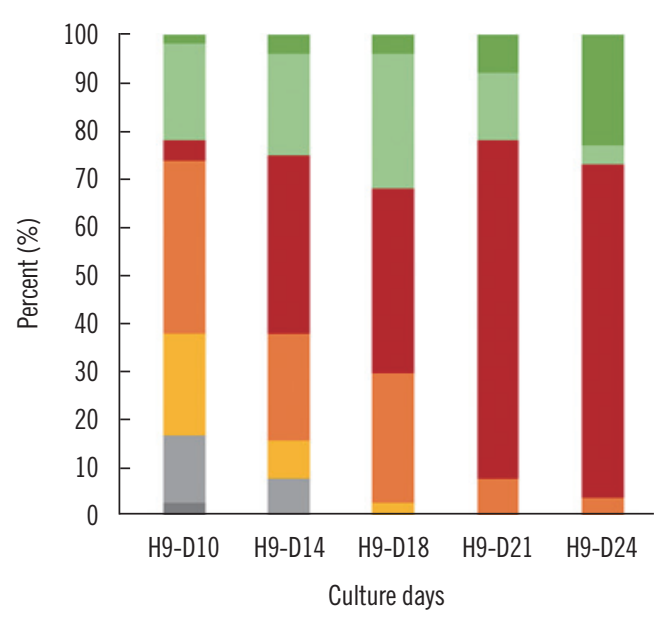

- Proerythroblast

- Orthochromatic erythroblast
Differential count of $\mathrm{H} 9$ cell

Early basophilic erythroblast

- Reticulocyte
Differential count of iPSC

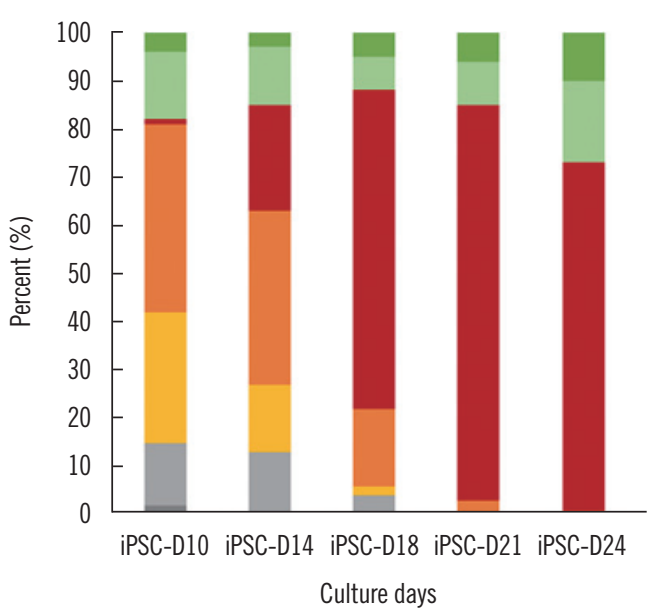

- Late basophilic erythroblast

- Granulocyte
- Polychromatic erythroblast

n Histiocyte

B

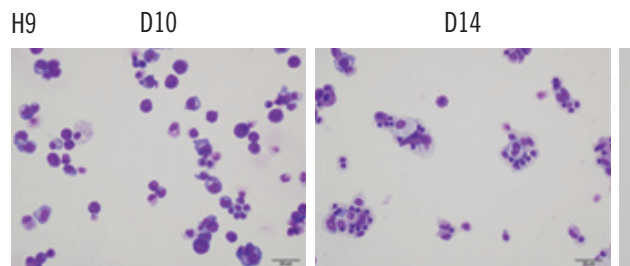

D18

D21
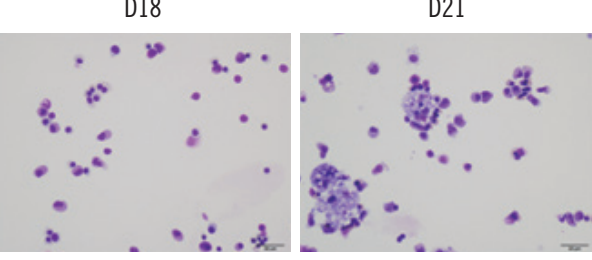

D24

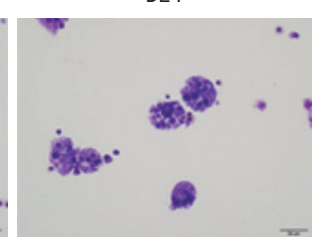

iPSC

D14

D18

D21

D24

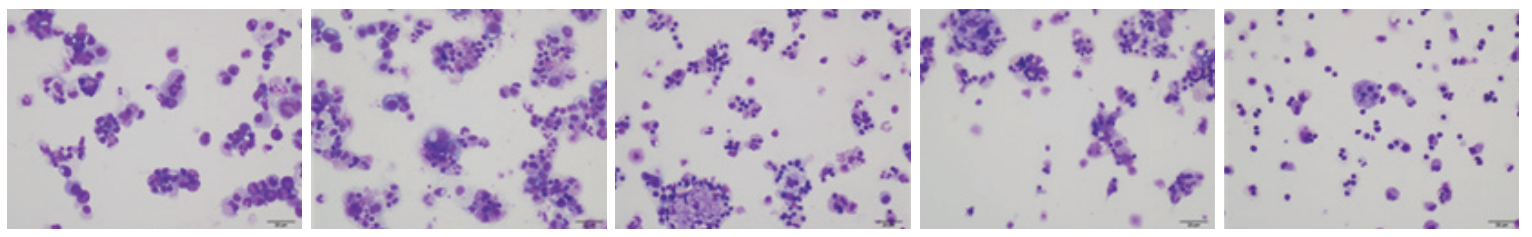

Fig. 4. (A) Differential counts of H9 cells (human embryonic stem cells) and iPSC cells revealed that differentiation caused a shift from hematopoietic stem cells to RBC precursors. (B) Photographs of cytospin preparations at different stages during erythropoiesis showing that the proportion of mature RBC precursors increases during the course of differentiation (Wright-Giemsa stain, $\times 400$, scale bar $=20 \mu \mathrm{m}$ ). Abbreviations: iPSC, induced pluripotent stem cell; RBC, red blood cell.

Blood group testing of cultured erythroid cells

The blood type of the donor with the highest yield of iPSC-derived erythroid cells was $O$ positive, and the iPSC-derived nRBCs were also $\mathrm{O}$ positive (A negative and $\mathrm{B}$ negative) (Fig. 6A). Moreover, iPSC-derived nRBCs exhibited the same $\mathrm{Rh}-\mathrm{Hr}$ subgroup phenotype as the donor, which was CcEe (Fig. 6B).

The pattern of blood group antigen expression of iPSC-derived nRBCs was the same as that of RBCs collected from the donor's blood, indicating that the iPSC-derived nRBCs expressed blood group antigens that could interact with blood group antibodies.

\section{DISCUSSION}

In vitro reagent RBC production may provide more consistent blood group antigen test results compared to direct blood donation. iPSCs represent an attractive source for RBC production because they are self-renewable and ethically acceptable [23]. Since iPSCs can be generated from various cell types [24], peripheral blood can be a useful source for iPSC production in a non-invasive manner [25]. We hypothesized that iPSC-derived nRBCs express blood group surface antigens capable of interacting with blood group antibodies. We obtained iPSCs from PBMNCs through transfection, which were then differentiated 
A

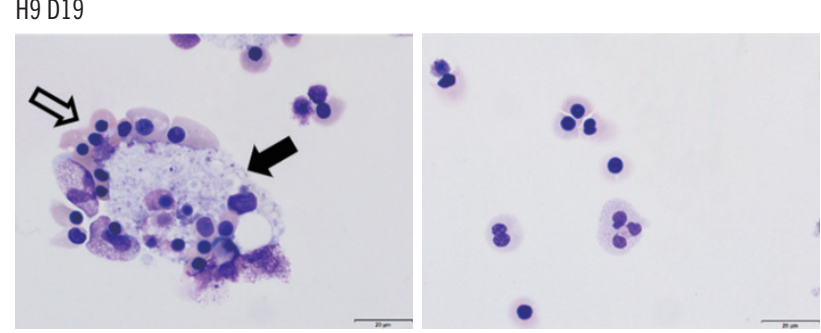

iPSC D19

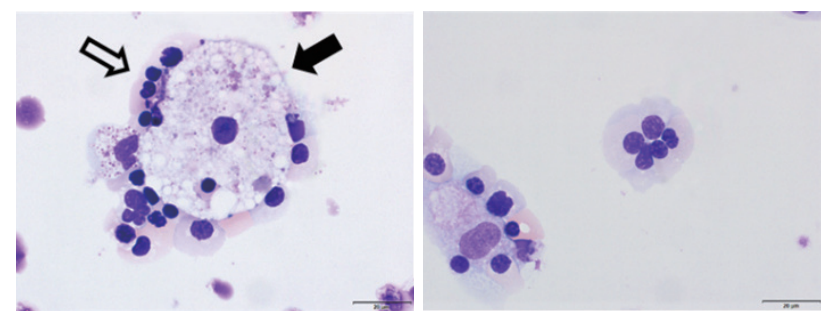

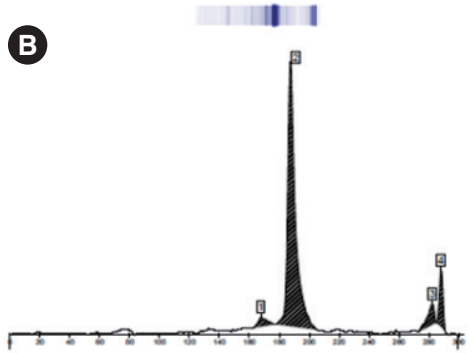

Hemoglobin electrophoresis

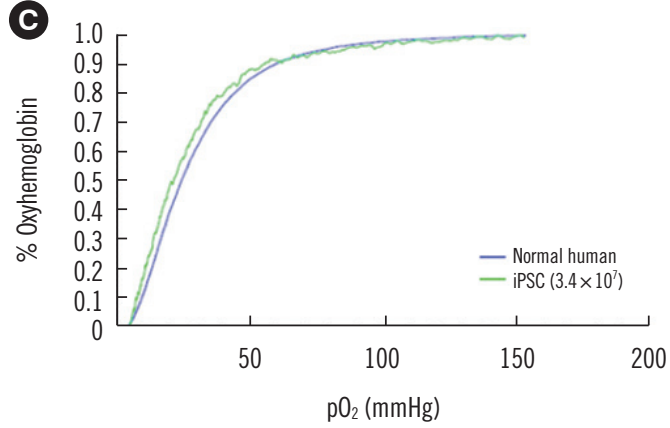

Fig. 5. Characterization of $n R B C s$ obtained from iPSCs. (A) nRBCs were observed on day 19 of the differentiation protocol. The nRBCs (white arrows) were observed to surround macrophages (black arrows); Wright-Giemsa stain, $\times 1,000$; scale bar=20 $\mu$ m. The human embryonic stem cell line $\mathrm{H} 9$ was counted as a control. (B) Hemoglobin electrophoresis analysis of iPSC-derived nRBCs showing a sharp peak in region 2, corresponding to $\mathrm{Hb} F$ based on comparison with the reference peak. (C) The oxygen-binding capacity did not differ between the healthy donor's RBCs (blue line) and iPSC-derived nRBCs (green line). Abbreviations: iPSC, induced pluripotent stem cell; nRBC, nucleated red blood cell.

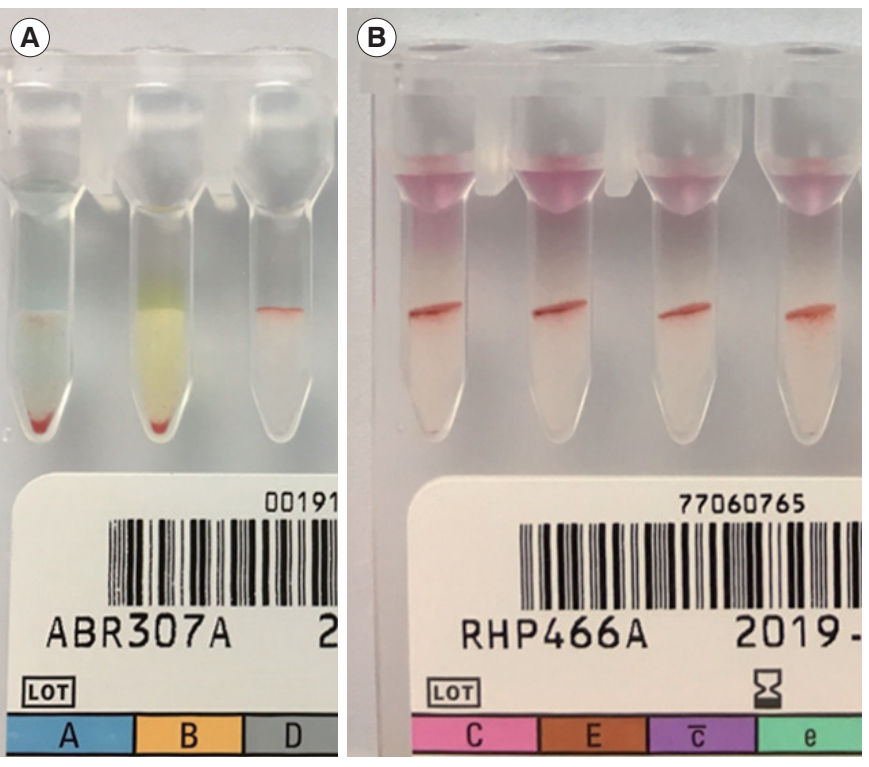

Fig. 6. Blood group testing of the nRBCs. The Ortho BioVue System Rh grouping cassette (Ortho Clinical Diagnostics, Raritan, NJ, USA) was used. (A) iPSC-derived $n R B C$ s did not react with anti-A and anti-B, whereas they reacted with anti-D, indicating that the blood group was O RhD-positive. (B) iPSC-derived nRBCs reacted with anti-C, anti-E, anti-C, and anti-e, indicating that the Rh-Hr phenotype was CcEe.

Abbreviations: iPSC, induced pluripotent stem cell; nRBC, nucleated red blood cell. into the erythroid lineage. On the last day of differentiation, the erythroid cells were treated with antibodies for $\mathrm{ABO}$ and $\mathrm{Rh}-\mathrm{Hr}$ blood grouping testing. We confirmed the same antigen expression pattern before (donor's PBMNCs) and after transfection (final product; nRBCs from iPSCs).

Retroviral, lentiviral, and episomal vector systems have been used to produce iPSCs [26]. Although retroviral and lentiviral vectors are relatively effective in forming colonies, they are more likely to undergo genomic mutations and cause cancer. Episomal vectors are not derived from viruses, but are unsuccessful in colony formation. We co-cultured iPSCs with OP9 bone marrow stromal cells during erythroid differentiation to improve the efficiency of the episomal vector system. The OP9 cell line was developed to induce the differentiation of mouse embryonic stem cells into myeloid, lymphoid, erythroid, and megakaryocytic lineage cells [16, 23, 27, 28]. Choi, et al. [29] used OP9 cells to differentiate hematopoietic cells from human fibroblastderived iPSC lines.

A major limitation of our study is that the cultured erythroid cells remained in the immature RBC state, which may explain the hemoglobin electrophoresis results showing the presence of fetal hemoglobin. Immature RBCs, including fetal RBCs, have been obtained after RBC production from iPSCs in most previ- 
ous studies [30-33]. Although blood group antigen expression has been observed in immature RBCs, mature cells are required for a robust reagent RBC development protocol [34]. Endothelial cells, which have the ability to produce erythroid progenitors with high enucleation potential, may provide a suitable microenvironment for erythroblasts to initiate terminal maturation [35]. The enucleation problem may be solved by adopting an endothelial cell-OP9 cell sequential cellular niche system. In addition to RBCs, macrophages have also been differentiated from hematopoietic stem cells, which are involved in RBC maturation by participating in the enucleation process [36]. Currently, we are developing a method for stimulating RBC maturation via the addition of more macrophages. In this study, iPSCs were co-cultured with OP9 mouse bone marrow stromal cells. At the beginning of differentiation, we separated the hematopoietic stem cells in the supernatant from the adherent OP9 cells; however, there is a possibility that residual OP9 cells may have contaminated the final product. The residual OP9 cells can be detected by flow cytometry using mouse-specific CD29 antibodies [37].

Improving RBC purity and viability is required for producing purer and more reliable diagnostic reagents. Fluorescence-activated cell sorting can be employed to improve cell purity; however, this technique requires expensive equipment and skilled technicians, which would increase the RBC production cost [38]. Cell viability was the highest on day 19 of differentiation, as the oxyhemoglobin dissociation curve for that day showed the least noise. It would be useful to track hemolysis trends by measuring free hemoglobin levels [39]. To enable the commercial application of reagent RBCs, the production process needs to be improved. For generating transfusable RBCs from iPSCs, they must be cultured in a serum- and feeder-free environment. This involves high production costs; specifically, cost-effectiveness imposes a limitation on the mass production of iPSC-derived RBCs for transfusion purposes. However, the production cost can be reduced when producing RBCs for diagnostic purposes.

We have presented a novel method for erythroid cell production using an OP9 cell co-culture protocol. This method enables producing nRBCs at low cost and is therefore suitable for diagnostic purposes. The serum-free, feeder-free culture 2D-multistep method proposed by Tursky, et al. [40] for generating $10^{6}$ progenitor cells costs $\$ 88.64$. In comparison, the OP9 co-culture method used in this study costs $\$ 38.29$. Although co-culture with OP9 cells lowers the development cost, artificial RBC generation is more expensive than RBC production through

blood donation. Lowering production costs would be a key factor in increasing the profitability of RBC production in vitro.

In conclusion, we established a protocol for erythroid differentiation of iPSCs co-cultured with OP9 cells. The produced nRBCs expressed blood group surface antigens that interacted well with blood group antibodies, and the oxygen-binding capacity of the produced nRBCs was similar to that of donated RBCs, indicating that the artificially generated erythroid cells can be used for the production of new RBC reagents for diagnostic purposes. Once iPSCs are established from the blood of rare blood group donors, iPSC-derived reagent RBCs can be produced when necessary. This study can contribute to obtaining reliable and consistent results in pretransfusion testing.

\section{ACKNOWLEDGMENTS}

We thank Eunkyung Lee and colleagues at the blood bank of Severance Hospital for their excellent technical assistance. We thank Bio-Rad (Aeran Kim, Field Application Supervisor, BioRad Laboratories Korea) for providing antibody identification cassettes. We thank Editage for language editing.

\section{AUTHOR CONTRIBUTIONS}

Roh $\mathrm{J}$ and $\mathrm{Kim} \mathrm{HO}$ designed the study. Jeon S-H and Kim H-K collected the data and searched the literature. Roh J and Kim $\mathrm{H}-\mathrm{K}$ analyzed the data and drafted the manuscript. Cheong J-W, Kim S, and Kim MJ revised the paper. All authors have read and approved the final manuscript.

\section{CONFLICTS OF INTEREST}

The authors have no conflicts of interest to declare.

\section{RESEARCH FUNDING}

This study was funded by the Severance Hospital Research Fund for Clinical Excellence (C-2019-0034).

\section{ORCID}

Juhye Roh

Sinyoung Kim June-Won Cheong

Su-Hee Jeon Hyun-Kyung Kim https://orcid.org/0000-0003-0078-1145 https://orcid.org/0000-0002-2609-8945 https://orcid.org/0000-0002-1744-0921 https://orcid.org/0000-0003-4558-4428 https://orcid.org/0000-0002-2528-6231 
Moon Jung Kim

https://orcid.org/0000-0003-4148-9116

Hyun Ok Kim

https://orcid.org/0000-0002-4964-1963

\section{REFERENCES}

1. Fung MK, Eder AF, Spitalnik SL, Westhoff CM. Technical Manual. 19th ed. Bethesda: American Association of Blood Banks, 2017.

2. Tormey CA and Hendrickson JE. Transfusion-related red blood cell alloantibodies: induction and consequences. Blood 2019;133:1821-30.

3. Bhuva DK and Vachhani JH. Red cell alloimmunization in repeatedly transfused patients. Asian J Transfus Sci 2017;11:115-20.

4. Olivier EN, Qiu C, Velho M, Hirsch RE, Bouhassira EE. Large-scale production of embryonic red blood cells from human embryonic stem cells. Exp Hematol 2006;34:1635-42.

5. Batta K, Menegatti S, Garcia-Alegria E, Florkowska M, Lacaud G, Kouskoff $V$. Concise review: recent advances in the in vitro derivation of blood cell populations. Stem Cells Transl Med 2016;5:1330-7.

6. Giarratana MC, Kobari L, Lapillonne H, Chalmers D, Kiger L, Cynober T, et al. Ex vivo generation of fully mature human red blood cells from hematopoietic stem cells. Nat Biotechnol 2005;23:69-74.

7. Douay $L$ and Giarratana MC. Ex vivo generation of human red blood cells: a new advance in stem cell engineering. Methods Mol Biol 2009; 482:127-40.

8. Chang TM. Evolution of artificial cells using nanobiotechnology of hemoglobin based RBC blood substitute as an example. Artif Cells Blood Substit Immobil Biotechnol 2006;34:551-66.

9. Chang TM. Hemoglobin-based red blood cell substitutes. Artif Organs 2004;28:789-94.

10. Shah S, Huang X, Cheng L. Concise review: stem cell-based approaches to red blood cell production for transfusion. Stem Cells Transl Med 2014;3:346-55.

11. Lee E, Sivalingam J, Lim ZR, Chia G, Shi LG, Roberts M, et al. Review: in vitro generation of red blood cells for transfusion medicine: progress, prospects and challenges. Biotechnol Adv 2018;36:2118-28.

12. Takahashi $\mathrm{K}$ and Yamanaka S. Induction of pluripotent stem cells from mouse embryonic and adult fibroblast cultures by defined factors. Cell 2006;126:663-76.

13. McLaren A. Ethical and social considerations of stem cell research. Nature 2001;414:129-31.

14. Clarke D and Frisén J. Differentiation potential of adult stem cells. Curr Opin Genet Dev 2001;11:575-80.

15. Gao J, Yan XL, Li R, Liu Y, He W, Sun S, et al. Characterization of OP9 as authentic mesenchymal stem cell line. J Genet Genomics 2010;37: 475-82.

16. Nakano T, Kodama H, Honjo T. Generation of lymphohematopoietic cells from embryonic stem cells in culture. Science 1994;265:1098-101.

17. Chen PK, Lin GL, Chang HH, Sun DS. Megakaryocytic differentiation of mouse embryonic stem cells via coculture with immortalized OP9 stromal cells. Exp Cell Res 2015;339:44-50.

18. Trakarnsanga K, Wilson MC, Heesom KJ, Andrienko TN, Srisawat C, Frayne J. Secretory factors from OP9 stromal cells delay differentiation and increase the expansion potential of adult erythroid cells in vitro. Sci Rep 2018;8:1983.

19. Vodyanik MA, Bork JA, Thomson JA, Slukvin II. Human embryonic stem cell-derived CD34+ cells: efficient production in the coculture with OP9 stromal cells and analysis of lymphohematopoietic potential. Blood 2005; 105:617-26.

20. Strober W. Trypan blue exclusion test of cell viability. Curr Protoc Immu- nol 2015;111:A3.B.1-a3.B.

21. Baker D, Hirst AJ, Gokhale PJ, Juarez MA, Williams S, Wheeler M, et al. Detecting genetic mosaicism in cultures of human pluripotent stem cells. Stem Cell Rep 2016;7:998-1012.

22. Hur M, Moon HW, Kwon SW. ABO-incompatible kidney transplantation. In: Ortiz J, ed. Understanding the complexities of kidney transplantation. 1st ed. IntechOpen, 2011: 332-48.

23. Dias J, Gumenyuk M, Kang H, Vodyanik M, Yu J, Thomson JA, et al. Generation of red blood cells from human induced pluripotent stem cells. Stem Cells Dev 2011;20:1639-47.

24. Revilla A, González C, Iriondo A, Fernández B, Prieto C, Marín C, et al. Current advances in the generation of human iPS cells: implications in cell-based regenerative medicine. J Tissue Eng Regen Med 2016;10: 893-907.

25. Dorn I, Klich K, Arauzo-Bravo MJ, Radstaak M, Santourlidis S, Ghanjati $\mathrm{F}$, et al. Erythroid differentiation of human induced pluripotent stem cells is independent of donor cell type of origin. Haematologica 2015; 100:32-41.

26. Nishino K, Arai Y, Takasawa K, Toyoda M, Yamazaki-Inoue M, Sugawara $\mathrm{T}$, et al. Epigenetic-scale comparison of human iPSCs generated by retrovirus, Sendai virus or episomal vectors. Regen Ther 2018;9:71-8.

27. Eto K, Murphy R, Kerrigan SW, Bertoni A, Stuhlmann H, Nakano T, et al. Megakaryocytes derived from embryonic stem cells implicate CalDAG-GEFI in integrin signaling. Proc Natl Acad Sci U S A 2002;99: 12819-24.

28. Nakano T, Kodama H, Honjo T. In vitro development of primitive and definitive erythrocytes from different precursors. Science 1996;272: $722-4$.

29. Choi KD, Yu J, Smuga-Otto K, Salvagiotto G, Rehrauer W, Vodyanik M, et al. Hematopoietic and endothelial differentiation of human induced pluripotent stem cells. Stem Cells 2009;27:559-67.

30. Kobari L, Yates F, Oudrhiri N, Francina A, Kiger L, Mazurier C, et al. Human induced pluripotent stem cells can reach complete terminal maturation: in vivo and in vitro evidence in the erythropoietic differentiation model. Haematologica 2012;97:1795-803.

31. Hansen M, Varga E, Aarts C, Wust T, Kuijpers T, von Lindern M, et al. Efficient production of erythroid, megakaryocytic and myeloid cells, using single cell-derived iPSC colony differentiation. Stem Cell Res 2018; 29:232-44.

32. Varga E, Hansen M, van den Akker E, von Lindern M. Erythropoiesis and megakaryopoiesis in a dish. In: Mehanna RA, ed. Cell culture. IntechOpen, 2018.

33. Lapillonne H, Kobari L, Mazurier C, Tropel P, Giarratana MC, ZanellaCleon I, et al. Red blood cell generation from human induced pluripotent stem cells: perspectives for transfusion medicine. Haematologica 2010;95:1651-9.

34. Southcott MJ, Tanner MJ, Anstee DJ. The expression of human blood group antigens during erythropoiesis in a cell culture system. Blood 1999;93:4425-35.

35. Shen J, Zhu Y, Lyu C, Feng Z, Lyu S, Zhao Y, et al. Sequential cellular niches control the generation of enucleated erythrocytes from human pluripotent stem cells. Haematologica 2020;105:e48-51.

36. de Back DZ, Kostova EB, van Kraaij M, van den Berg TK, van Bruggen R. Of macrophages and red blood cells; a complex love story. Front Physiol 2014;5:9.

37. Vodyanik MA and Slukvin II. Hematoendothelial differentiation of human embryonic stem cells. Curr Protoc Cell Biol 2007; Chapter 23:Unit 23.6.

38. Basu S, Campbell HM, Dittel BN, Ray A. Purification of specific cell population by fluorescence activated cell sorting (FACS). J Vis Exp 
2010;1546.

39. Gladwin MT, Kanias T, Kim-Shapiro DB. Hemolysis and cell-free hemoglobin drive an intrinsic mechanism for human disease. J Clin Invest 2012;122:1205-8.
40. Tursky ML, Loi TH, Artuz CM, Alateeq S, Wolvetang EJ, Tao H, et al. Direct comparison of four hematopoietic differentiation methods from human induced pluripotent stem cells. Stem Cell Rep 2020;15:735-48. 
Roh J, et al.

A
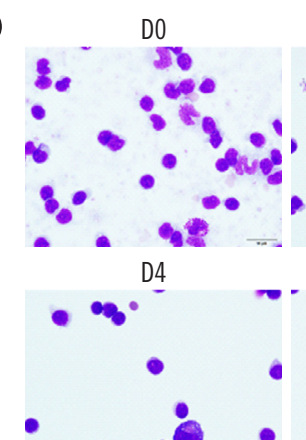

-

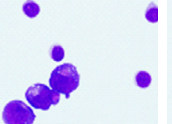

D1

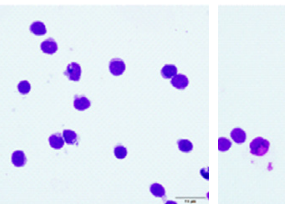

D5

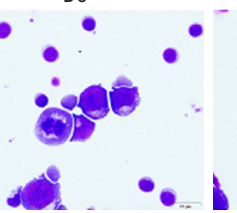

D2
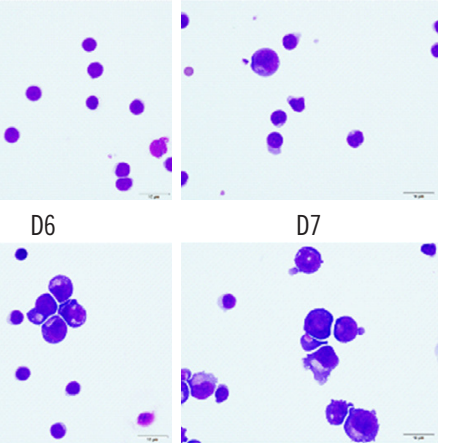

B

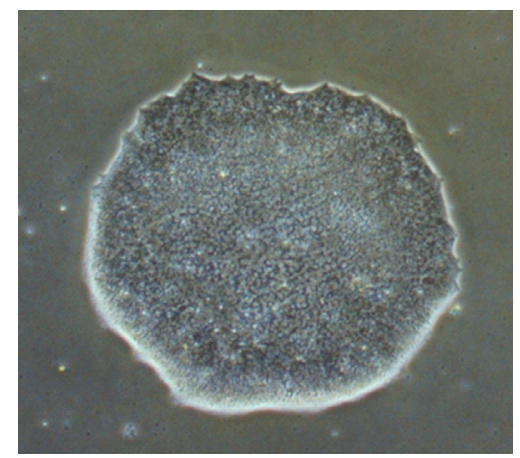

Supplemental Data Figure S1. Generation of iPSCs from PBMNCs. (A) During the expansion step, erythroblasts were counted to determine the optimal time point for transfection. When the erythroblast population reached $80 \%$ of the total cell population, which occurred on day 7 , the cells were transfected (Wright-Giemsa stain, $\times 1,000$; scale bar $=10 \mu \mathrm{m}$ ). (B) After transfection, the cells clumped together into small iPSC colonies $(\times 40)$.

Abbreviations: IPSC, induced pluripotent stem cell; PBMNC, peripheral blood mononuclear cell. 